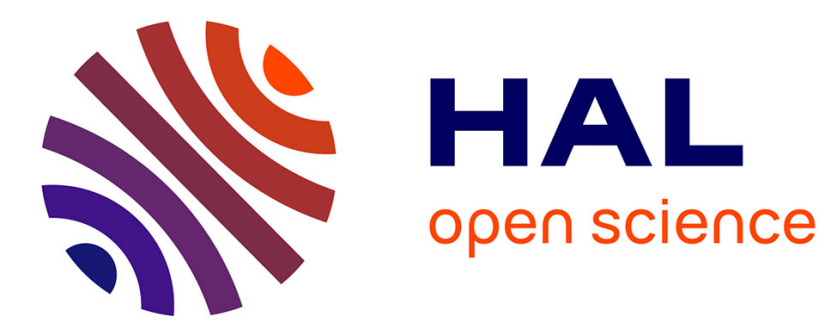

\title{
Le monde va trop vite pour les personnes autistes! Hypothèses neurophysiopsychopathogéniques et implications rééducatives \\ Bruno Gepner
}

\section{To cite this version:}

Bruno Gepner. Le monde va trop vite pour les personnes autistes! Hypothèses neurophysiopsychopathogéniques et implications rééducatives. Neuropsychiatrie de l'Enfance et de l'Adolescence, 2006, 54, pp.371-374. hal-00261586

\section{HAL Id: hal-00261586 \\ https://hal.science/hal-00261586}

Submitted on 7 Mar 2008

HAL is a multi-disciplinary open access archive for the deposit and dissemination of scientific research documents, whether they are published or not. The documents may come from teaching and research institutions in France or abroad, or from public or private research centers.
L'archive ouverte pluridisciplinaire HAL, est destinée au dépôt et à la diffusion de documents scientifiques de niveau recherche, publiés ou non, émanant des établissements d'enseignement et de recherche français ou étrangers, des laboratoires publics ou privés. 


\section{Référence de l'article :}

Gepner, B. (2006). Le monde va trop vite pour les personnes autistes ! Hypothèses neurophysio-psychopathogéniques et implications rééducatives. Neuropsychiatrie de l'Enfance et de l'Adolescence, 54, 371-374.

\section{Le monde va trop vite pour les personnes autistes ! Hypothèses neurophysiopsychopathogéniques et implications rééducatives}

\section{B. Gepner}

Service de psychiatrie infanto-juvénile, Centre Hospitalier Montperrin, 109, avenue du Petit Barthélémy 13090 Aix-en-Provence

Adresse e-mail : bruno.gepner@lpl.univ-aix.fr (B. Gepner)

\section{Résumé}

Le monde environnemental va trop vite pour certaines personnes souffrant d'autisme !

Ainsi, les enfants autistes ont du mal à percevoir et intégrer les mouvements visuels, qu'il s'agisse de mouvements physiques (flux visuel, mouvement cohérent, mouvement de points singuliers) ou de mouvements dits «biologiques » (c'est-à-dire les mouvements d'êtres vivants, comme les mouvements corporels ou les mouvements faciaux, ceux des yeux et des lèvres et les mimiques émotionnelles). Cette difficulté est, semble-t-il, d'autant plus prononcée que les mouvements sont rapides et que la pathologie des enfants est importante (CARS élevée, retard de langage ou retard mental associé). Similairement, certains enfants autistes ont un trouble de la catégorisation de certains phonèmes ambigus lorsque ceux-ci leur sont présentés en parole normale, et leur catégorisation se normalise en parole ralentie.

Le traitement temporel des mouvements visuels et du flux sonore serait donc défectueux chez les enfants avec autisme. Autrement dit, leur sensorialité et leur sensorimotricité seraient marquées par un défaut de traitement en ligne des événements visuels et auditifs, ainsi que d'un défaut de couplage sensorimoteur en temps réel.

Récemment, notre groupe vient de montrer que le ralentissement de mimiques faciales émotionnelles et non émotionnelles et de leur son correspondant améliorait la reconnaissance de ces mimiques et en induisait l'imitation faciale et vocale, chez des enfants autistes, et plus particulièrement chez ceux dont le degré d'autisme est le plus sévère.

L'hypothèse neurofonctionnelle d'un trouble du codage temporel et de la synchronisation neuronale sous-jacent à ce défaut de traitement temporel, et la question d'une parenté physiopathogénique entre autisme, troubles de développement du langage et schizophrénie seront discutées. Nous évoquerons également les perspectives de recherche rééducative qui découlent logiquement de nos résultats.

Mots clés : Autisme ; Mouvements physiques ; Mouvements biologiques ; Mimiques faciales émotionnelles ; Dysynchronie ; Dysconnectivité ; Ralentissement 


\title{
Title
}

The world is going too fast for individuals with autism ! Neurophysiopathogenic hypotheses and reeducational consequences

\begin{abstract}
The environmental world is going too fast for some persons with autism !

Indeed, some children with autism have difficulties in perceiving and integrating visual movements, physical movements (optic flow, coherent motion) as well as biological ones (body movements, facial movements such as movements of the lips, movements of the eyes, or emotional gestures). These difficulties appear to be more pronounced when movements are rapid or when pathology of the children is severe (high degree of autism, association with mental or verbal delay). Similarly, some children with autism are impaired in categorizing ambiguous phonemes when displayed at a normal speed, and their impairment is normalized when phonemes are displayed slowly.

Therefore, the temporal processing of visual movements and verbal flow is likely to be impaired in children with autism. In other words, their visual and auditory online processing would be defectuous, as well as their real-time sensorymotor coupling.

Recently our group has demonstrated that when the speed of facial movements and their corresponding vocal sounds is slowed down, recognition and induced imitation of emotional and non-emotional facial expressions are enhanced in children with autism, especially in those having higher degrees of autism.

We propose that these sensory processing peculiarities in autism may be explained by a deficit in temporal coding and neural synchronization. We also underline the physiopathogenic similarities between autism and language learning impairments, and even with schizophrenia. We finally suggest the logical consequences of our approach for reeducation of people with autism.
\end{abstract}

Key words : Autism; physical movements; biological movements; emotional facial expressions ; dyssynchrony ; dysconnectivity ; slowing down

Au cours de cette conférence de juin 2005 au congrès de la Société française de psychiatrie de l'enfant et de l'adolescent et disciplines associées (SFPEADA), nous avons poursuivi la description de notre approche neuropsychologique développementale des désordres du spectre autistique, centrée sur des difficultés à percevoir et intégrer les mouvements physiques de l'environnement, mais aussi les mouvements biologiques tels que les mouvements faciaux et, en particulier, les mimiques faciales émotionnelles. Nous résumons cette approche par le concept de «malvoyance » du mouvement [1] et maintenant celui de « malvoyance de l'é-motion » (qui contient la notion de désordre émotionnel et motionnel). Nous avons rappelé les différents arguments cliniques (notamment ceux issus des films familiaux, voir Sauvage, L'autisme du nourrisson, 1988), autobiographiques (Temple Grandin, Donna Williams, Van Dalen) et expérimentaux en faveur de cette «malvoyance de l'é-motion » dans l'autisme. Nous avons insisté sur le fait que la vitesse du mouvement semblait constituer un facteur nodal dans l'autisme, autrement dit que le monde visuel va trop vite pour au moins certaines personnes autistes, certains enfants autistes semblant d'autant plus gênés que la vitesse des stimuli sensoriels est élevée [2]. Selon cette approche, les défauts de perception visuelle et d'intégration visuomotrice du mouvement physique environnemental $[3,4]$ pourraient rendre compte des anomalies précoces dans le 
développement de l'anticipation motrice, de l'ajustement visuopostural et de la posturomotricité. Les défauts de perception visuelle et d'intégration visuomotrice des mouvements biologiques humains, corporels, gestuels et faciaux, pourraient quant à eux rendre compte de leurs problèmes de lecture des visages, d'imitation faciale et corporelle, de communication verbale et émotionnelle et d'interaction socio-affective, qui apparaissent donc secondaires aux désordres perceptifs [5]. Ces défauts perceptifs et intégratifs pourraient enfin expliquer les difficultés d'accès à la conscience de la continuité et de l'unité corporelle chez certains enfants autistes [6,7].

Nous avons complété notre exposé en montrant que la malvoyance du mouvement rapide était un exemple particulier, mais pas le seul, d'anomalies du traitement des événements environnementaux dans l'autisme. En effet, certains événements sonores rapides (des phonèmes ambigus) sont mal catégorisés [8] par des enfants autistes, et certains événements proprioceptifs sont traités de manière ralentie, selon des modalités rétroactives et non proactives, par ces mêmes enfants autistes [9]. Les enfants autistes souffrent aussi de troubles du traitement des fréquences spatiales basses impliquées dans des stimuli visuels faciaux et non faciaux [10]. Ainsi, les personnes autistes souffriraient de troubles plus globaux du traitement temporospatial, c'est-à-dire d'anomalies dans la perception temporelle et spatiale en ligne des événements sensoriels en provenance du monde environnemental, ainsi que dans le couplage sensorimoteur et la production en temps réel d'événements moteurs et de communication verbale et émotionnelle, et ce, depuis les débuts de leur vie post-natale (Figure 1) [11,12].

Puis, nous avons envisagé les différents modèles neurophysiopathologiques susceptibles de rendre compte de ces troubles du traitement temporel dans l'autisme: troubles du codage temporel dans chaque sous-système sensoriel et sensori-moteur, en partie liés à un dysfonctionnement cérébelleux (l'horloge interne du cerveau) [13,14]; réduction de la perception cohérente du monde par simultanéité [15], qui renforcerait la théorie du déficit de cohérence centrale de Frith [16]; Temporal binding deficit hypothesis, c'est-à-dire réduction de la synchronisation des décharges neuronales de différents réseaux neuronaux locaux spécialisés, liées aux ondes gamma [17,18] (mais Brown et al. [19] montrent un résultat contraire à la prédiction, c'est-à-dire une augmentation de la synchronisation gamma dans une tâche de perception de formes) ; désordres de connectivité neuronale [20], avec hyperconnectivité locale et hypoconnectivité à distance [21].

Il faut retenir de ces travaux et hypothèses convergents l'idée que la connectivité spatiale entre différents systèmes neurofonctionnels ou la synchronisation temporelle entre ces différents systèmes sont altérées dans l'autisme. De multiples facteurs peuvent concourir seuls ou ensemble à altérer cette connectivité/synchronisation intracérébrale : défaut de connectivité structurale, liée à des facteurs génétiques [22] ou épigénétiques [23] ; altération de la connectivité structurale ou fonctionnelle par des facteurs infectieux, métaboliques ou immunitaires affectant un ou plusieurs territoires neurofonctionnels, puis de proche en proche, par effet «tâche d'huile » ou «boule de neige », altération de la connectivité cérébrale globale.

Nous avons proposé le concept de dys-synchronie et dys-connectivité fonctionnelle multisystème, pour rendre compte de ces anomalies rencontrées dans l'autisme, le préfixe dys-, voulant dire que les sujets autistes peuvent souffrir d'hypo- ou d'hyper-synchronisation neuronale, et d'hypo- ou hyper-connectivité neuronale [6,7,12]. En particulier, il est concevable que l'hyper-connectivité et l'hyper-synchronisation neuronale soit un moyen de compenser une hypo-connectivité ou désynchronisation préalable, L'épilepsie (fréquemment comorbide de l'autisme) serait une manifestation extrême de cette hypersynchronisation.

Les difficultés des personnes autistes à percevoir les événements ou flux sensoriels en ligne 
(c'est-à-dire au moment où ils leur parviennent), à intégrer ces flux dans leur corps propre, à coupler en temps réel perception et action, et à s'accorder cognitivement et émotionnellement à autrui dans les échanges communicatifs et sociaux pourraient ainsi être une traduction comportementale et neuropsychologique de cette dys-synchronie et dysconnectivité neurobiologique, que celle-ci soit d'ordre structural ou fonctionnel.

En accord avec l'hypothèse de Welsh et al. [24], il est vraisemblable que certains événements sensoriels (notamment les flux visuels, sonores et tactilo-kinesthésiques rapides, ou les changements environnementaux physiques et biologiques rapides) surviennent et parviennent trop vite à certaines personnes autistes, et débordent leurs capacités cérébrales de synchronisation, et du coup désynchronisent les décharges neuronales au sein d'un système et entre plusieurs systèmes, altèrent la connectivité intracérébrale et produisent des cascades mal-développementales au sein de nombreux mécanismes neuropsychodéveloppementaux, de manière plus ou moins envahissante. Une hyper-synchronisation pourrait, quant à elle, survenir soit d'emblée pour certains stimuli (statiques notamment), soit secondairement à une désynchronisation, pour en compenser les effets néfastes, et dépasser parfois ses objectifs sous la forme d'hyper-synchronisation pathologique et d'épilepsie [6,7,29].

Pour rendre compte de la parenté de certains signes cliniques présents dans les désordres autistiques d'un côté, et les syndromes dys- (dysphasie, dyslexie, dyspraxie...), les troubles attentionnels avec ou sans hyperactivité et les syndromes épileptiques, d'un autre côté, nous avons également suggéré que ces divers troubles neurodéveloppementaux partageaient sans doute au moins un mécanisme physiopathogénique commun. Nous proposons que ce mécanisme physiopathogénique commun, permettant d'expliquer cette tendance à la comorbidité et à des recouvrements nosographiques entre ces différents syndromes, relève de la synchronisation-connectivité neuronale. En effet, l'existence de troubles du traitement temporel a été démontrée il y a 30 ans chez certains patients atteints de troubles de développement du langage (dysphasie, dyslexie) [25], des désordres de la connectivité cérébrale ont été démontrés chez des sujets atteints de troubles attentionnels, et les syndromes épileptiques sont marqués par des troubles de la synchronisation neuronale. Mais, des troubles de la connectivité et de la synchronisation sont également à l'œuvre dans la schizophrénie [26] et la dépression.

Que conclure de ceci ? Aussi différents soient-ils sur le plan clinique, les troubles neurodéveloppementaux, ou les différentes maladies neuropsychiques, pourraient comporter dans leurs soubassements neurobiologiques fondamentaux, une altération de la synchronisation-connectivité neuronale. Cette altération, en fonction de ses origines, de sa nature, de son intensité, de ses localisations, de son extension temporospatiale, se manifesterait par des expressions phénotypiques variées, le long d'un continuum clinique.

Cette vision relativise, c'est-à-dire met en relation, l'autisme par rapport aux autres désordres neuropsychiques, ce qui ne doit pas empêcher (bien au contraire) de continuer de spécifier les différences entre les divers désordres du spectre autistique, et, entre ceux-ci et les autres désordres complexes neurodéveloppementaux et neuropsychiques, afin d'ouvrir de nouvelles fenêtres de compréhension et de traitement plus spécifiques de ces différents désordres.

Pour finir, nous avons présenté une application clinique de notre théorie de la «malvoyance de l'é-motion» et autres troubles du traitement temporospatial des flux sensoriels. Nous avons en particulier présenté les résultats d'une étude confirmant des résultats antérieurs [27] et qui montre que le ralentissement de mimiques faciales émotionnelles et non-émotionnelles et de leurs sons correspondants améliore la 
reconnaissance de ces mimiques sur photographie, et induit une imitation faciale et vocale de ces mimiques $[28,29]$. Ce résultat constitue une validation de notre approche et pourrait, à l'avenir, ouvrir de nouvelles perspectives rééducatives. Nous sommes actuellement en train de tester l'impact d'un logiciel de ralentissement simultané des mouvements faciaux et de la parole sur la compréhension verbale et l'imitation d'enfants et adolescents autistes, retardés mentaux, et témoins ordinaires. Ce ralentissement des événements environnementaux pourrait faciliter la synchronisation neuronale dans le cerveau des personnes autistes, et constituer, de facto, un facteur synchronisant [6,7,29].

\section{RÉFÉRENCES}

[1] Gepner B. «Malvoyance » du mouvement dans l'autisme infantile ? Une nouvelle approche neuropsychopathologique développementale. La Psychiatrie de l'Enfant 2001; 1: 77-126.

[2] Gepner B, Mestre D. Rapid visual-motion integration deficit in autism. Trends in Cognitive Sciences 2002; 6:11, 455.

[3] Gepner, B., Mestre, D., Masson, G., de Schonen, S. Postural effects of motion vision in young autistic children. NeuroReport, 1995; 6: 1211-1214.

[4] Gepner B, Mestre D. Postural reactivity to fast visual motion differentiates autistic from children with Asperger syndrome. Journal of Autism and Developmental Disorders 2002; 32: 231-8.

[5] Gepner B. Autism, movement and facial processing. The American Journal of Psychiatry 2004; 161: 1719.

[6] Gepner B, Tardif C. Autism, movement, time and tought. In: Frontiers in Cognitive Psychology, Colombus F, ed. New York: Nova Science publishers, in press.

[7] Gepner B. Constellation autistique, mouvement, temps et pensée. Devenir, in press.

[8] Tardif C, Thomas K, Gepner B, Rey V. Contribution à l'évaluation du système phonologique explicite chez des enfants autistes. Parole 2002; 21: 35-72.

[9] Schmitz C, Assaiante C, Gepner B. Modulation de la réponse anticipée en fonction du poids à délester : étude chez l'enfant sain et l'enfant autiste. TIPA 2002; 21: 207-11.

[10] Deruelle C, Rondan C, Gepner B, Tardif C. Spatial frequency and face processing in children with autism and Asperger syndrome. Journal of Autism and Developmental Disorders 2004; 34: 199-210.

[11] Gepner B. Malvoyance du mouvement dans l'autisme : de la clinique à la recherche et à la rééducation. In : L'Autisme : de la recherche à la pratique, Andrès C, Barthélémy C, Berthoz A, Massion J, Rogé B, eds. Paris : Odile Jacob ; 2005. p. 205-226.

[12] Gepner B, Lainé F, Tardif C. E- Motion mis-sight and other temporal processing disorders in autism. Current Psychology of Cognition-Cahiers de psychologie cognitive $2005 ; 23: 104-21$.

[13] Johnson MT, Ebner TJ. Processing of multiple kinematic signals in the cerebellum and motor cortices. Brain Research Review 2000; 33: 153-68.

[14] Gepner B, Massion J. L'autisme : une pathologie du codage temporel ? TIPA 2002; 21: 177-218.

[15] Berthoz A (1997). Le sens du mouvement. Paris : Odile Jacob.

[16] Frith U. Autism: explaining the enigma. Basic Blackwell; 1989.

[17] Varela F, Lachaux JP, Rodriguez E, Martinerie J. The Brainweb: phase synchronization and largescale integration. Nature Reviews Neuroscience 2001; 2: 229-39.

[18] Brock J, Brown CC, Boucher J, Rippon G. The temporal binding deficit hypothesis of autism. Developmental Psychopathology 2002; 14: 209-24.

[19] Brown C, Gruber T, Boucher J, Rippon G, Brock J. Gamma abnormalities during perception of illusory figures in autism. Cortex 2005; 41: 364-76.

[20] Wickelgren I. Autistic brains out of synch? Science 2005; 308: 1856-58.

[21] Courchesne E, Pierce K. Why the frontal cortex in autism might be talking only to itself: local overconnectivity but long-distance disconnection. Current Opinion in Neurobiology 2005; 15: 225-30.

[22] Jamain S, Quach H, Betancur C, Rastam M, Colineaux C, Gillberg IC, et al. Mutations of the X-linked genes encoding neuroligins NLGN3 and NLGN4 are associated with autism. Nature Genetics 2003; 34: $27-9$.

[23] Gepner B, Soulayrol R. Utilité des concepts d'épigenèse et d'auto-organisation pour la compréhension des syndromes autistiques de l'enfant. La Psychiatrie de l'Enfant 1994; 1: 115-52.

[24] Welsh JP, Ahn ES, Placantonakis DG. Is autism due to brain desynchronization? International Journal of Developmental Neuroscience 2005; 23: 253-63.

[25] Tallal P. Rapid auditory processing in normal and disordered language development. Journal of Speech \& Hearing Research 1976; 19: 561-94.

[26] Spencer KM, Nestor FG, Perlmutter R, Niznikiewicz MA, Klump MC, Frumin M, et al. Neural synchrony indexes disordered perception and cognition in schizophrenia. Proceedings of the National 
Academy of Sciences 2004; 101: 7288-93.

[27] Gepner B, Deruelle C, Grynfeltt S. Motion and emotion: a novel approach to the study of face processing by autistic children. Journal of Autism and Developmental Disorders 2001; 31: 37-45.

[28] Lainé F, Tardif C, Gepner B. Amélioration de la reconnaissance et de l'imitation d'expressions faciales chez des enfants autistes grâce à une présentation visuelle et sonore ralentie. Annales médico-psychologiques, in press.

[29] Tardif C, Lainé F, Rodriguez M, Gepner B. Slowing down facial movements enhances facial expression's recognition and imitation in autistic children. Journal of Autism and Developmental Disorders, in press.

Figure 1. Schéma synthétique des cascades 'maldéveloppementales' de désordres du traitement temporospatial des événements sensoriels dans l’autisme (repris de Gepner, 2005)

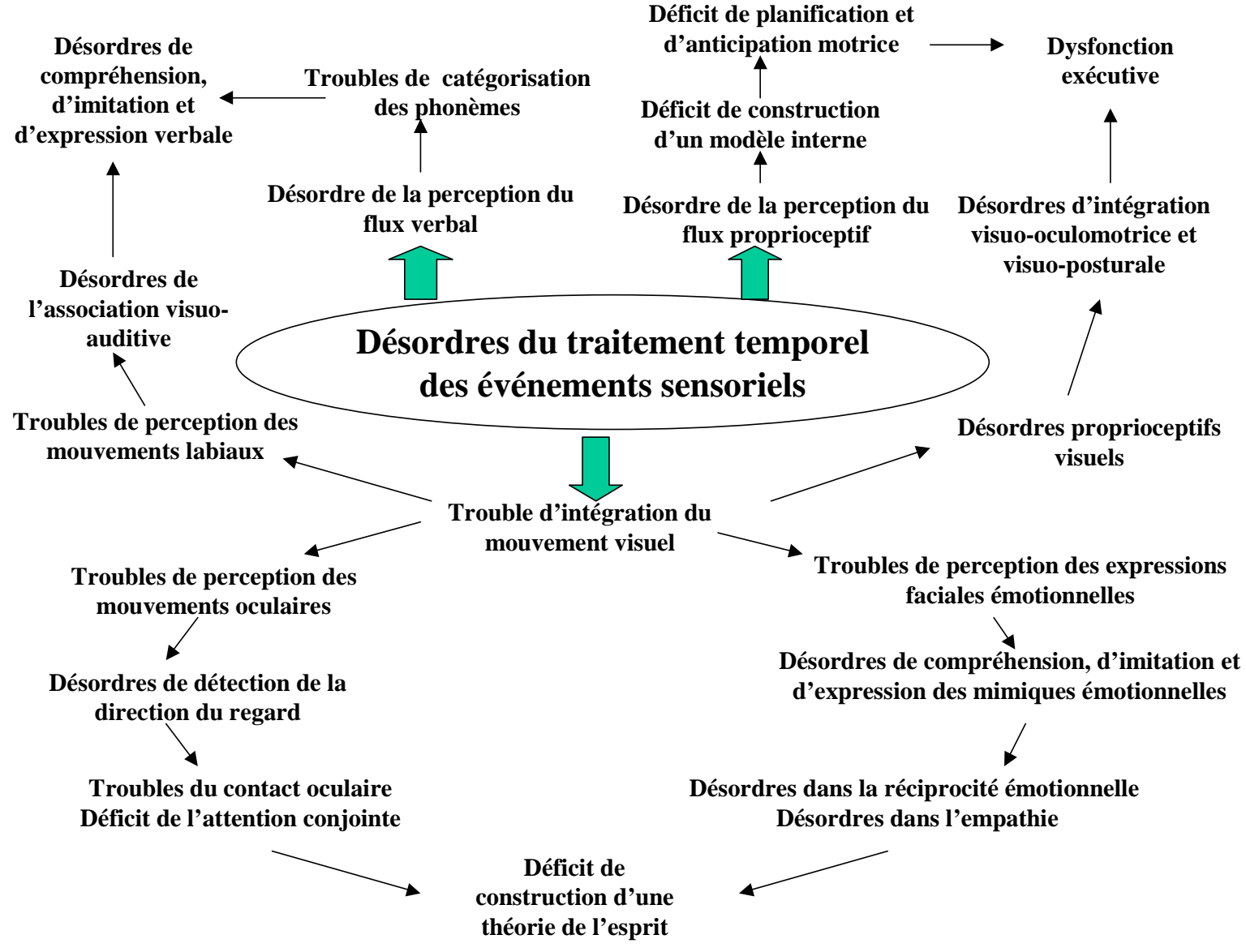

